\title{
Assessing immunization programs
}

\author{
Joanne Embree MD FRCPC
}

$\mathrm{O}$ ur ability to prevent or modify disease through immunization has expanded dramatically over the past few decades, and there is incredible pressure to continue to develop and quickly utilize new vaccines. This is due to the recognition that the prevention of diseases is more cost effective than the treatment of them, and that the effective immunization programs that are currently in place have proven to be among the most successful public health measures.

The process of developing and evaluating vaccine efficacy and safety before licensure is stringent. However, there are limitations to these procedures. All potential adverse events may not be apparent with the relatively limited number of individuals who take part in the prelicensure studies. Thus, following licensure, there are a number of systems in place for the ongoing monitoring of vaccine safety. That these systems are effective has been demonstrated recently by the withdrawal of the rotavirus vaccine from the American market within months of licensure, following the discovery of an association between vaccine administration and a transient increased risk of intussusception (1). Canada monitors postlicensure vaccine safety in a number of ways (2). There is a passive reporting system (the Vaccine Associated Adverse Events Surveillance System) that is used by health care providers and vaccine recipients (or their care givers). This is coupled with a formal assessment conducted by the Advisory Committee on Causality Assessment of the likelihood that a particular vaccine caused any reported serious or unexpected adverse event. In addition, there are two active surveillance systems that monitor vaccine safety for children. Both are collaborative efforts between Health Canada and the Canadian Paediatric Society. The Immunization Monitoring Program ACTive (IMPACT) is a hospital-based surveillance system. The medical charts of children who are admitted to any of the paediatric hospitals that participate in the IMPACT surveillance system are reviewed for targeted potential adverse events related to recent vaccine administration and for the occurrence of vaccine preventable diseases. The
Canadian Paediatric Surveillance Network, in which paediatricians across the country report cases of rare illnesses targeted for national surveillance, also has the capacity to monitor for rare events associated with recent immunizations. This system is not dependent on admission to hospital. The direct effect of the introduction of new immunization programs on the incidences of the diseases that the new programs are designed to prevent, however, is not assessed systematically in Canada.

The introduction of a new vaccine or immunization program is expected to reduce the disease burden caused by the pathogen against which the vaccine is directed. In Canada, this effect is difficult to measure directly for a number of reasons. Immunization programs are introduced sporadically, province by province. Therefore, the effect of the introduction of these programs on nationally summarized data on disease incidence is difficult to interpret. Individuals move and travel frequently, and infectious illnesses do not generally respect provincial borders. Thus, an immunization program in one province may lower the disease incidence in another province that does not provide the vaccine. Alternatively, a program may not be as effective as anticipated because of the continuous introduction of the illness into the population from neighbouring provinces or territories. Currently, immunization uptake is estimated from vaccine sales or distribution, which may not accurately reflect usage. There is also no way for public health departments to easily link vaccine administration and disease illness to determine whether the occurrence of vaccine-preventable disease in individuals or groups is related to missed immunization opportunities or to primary vaccine failure. To do this, specific studies must be financed and undertaken. In this era of doubt that is increasingly being expressed by some members of the public concerning the usefulness and safety of vaccination, it is important that we have a more systematic method of assessing the immediate impact of the introduction of new immunization programs on the burden of disease.

Associate Editor-in-Chief, University of Manitoba, Winnipeg, Manitoba

Correspondence: Dr Joanne Embree, Basic Medical Sciences Building, 730 William Avenue, Room 530, Winnipeg, Manitoba

R3E OW3. Telephone 204-789-3630, fax 204-789-3926, e-mail embree@ms.umanitoba.ca 
However, we also need to assess the long term effects of the introduction of immunization programs. The development of sophisticated modelling techniques and their acceptance as useful scientific tools have greatly enhanced our ability to predict the effect of public health interventions. The usefulness of any model is obviously very dependent upon the data available and the assumptions that are made to produce it. Small changes in the assumptions used in modelling can have a significant effect on the predicted outcome. Despite this limitation, this type of research is becoming an essential part of health program planning. The development of systematic surveillance systems to monitor for the modelled outcomes when immunization programs are introduced based on the conclusions from the models is necessary. This is particularly important when the vaccine may not provide lifelong immunity and continued protection from disease among vaccine recipients, and is dependent on continued exposure to the pathogen. Temporary elimination of the pathogen in the population due to an effective immunization program among children may result in a substantial proportion of the population becoming susceptible later in life. If the disease is one in which older individuals suffer significantly more morbidity or mortality than do children, or in which there is concern for significant congenital abnormalities with primary infection during pregnancy, there may paradoxically be a substantial overall increase in disease burden in the future. To address these concerns, formalized modelling of the various patterns of disease incidence that could potentially occur is invaluable. Coupled with this activity, however, is the need to commit resources for ongoing testing and reassessment of the models that are produced. As new information becomes available through systematic surveillance of disease incidence, the accuracy of the predictions from the models can be better evaluated. More importantly, public health officials will then be able to adequately make and justify decisions concerning additional interventions, such as the need for the introduction of serological screening in pregnancy or adult immunization programs.

It is extremely important that we acknowledge fully the possibility of either early or long term consequences with the introduction of new immunization programs or vaccines, actively monitor for them, and formulate potential solutions in advance of their occurrence. This should be one of the objectives of a national immunization strategy for Canada.

\section{REFERENCES}

1. Delage G. Rotavirus vaccine withdrawal in the United States: The role of postmarketing surveillance. Can J Infect Dis 2000;11:10-2.

2. Embree JE, Infectious Diseases and Immunization Committee of the Canadian Paediatric Society. It's time for a national immunization strategy. Can J Infect Dis 2001;12:208-10. 


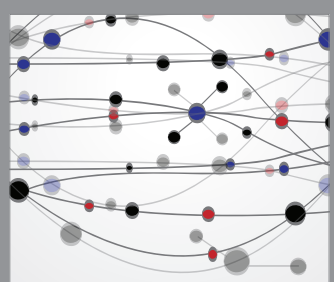

The Scientific World Journal
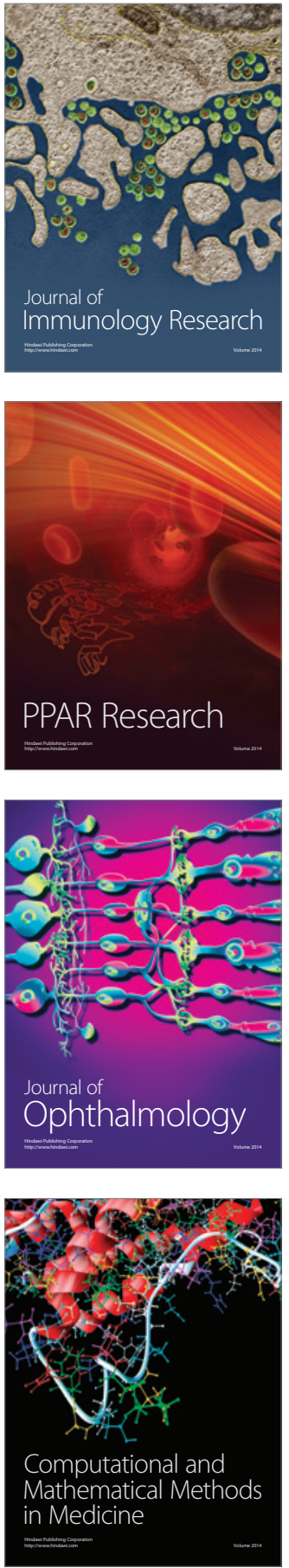

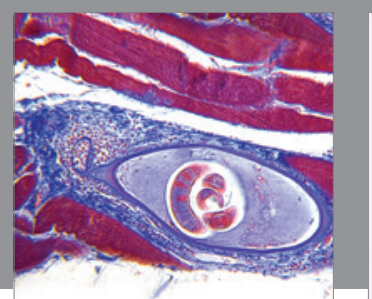

Gastroenterology Research and Practice

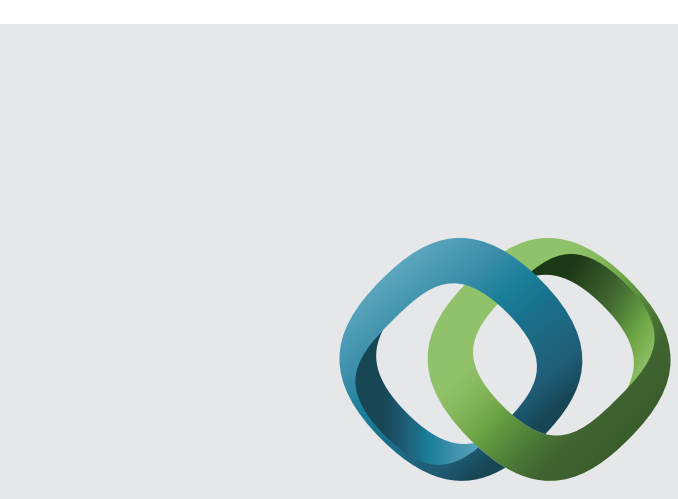

\section{Hindawi}

Submit your manuscripts at

http://www.hindawi.com
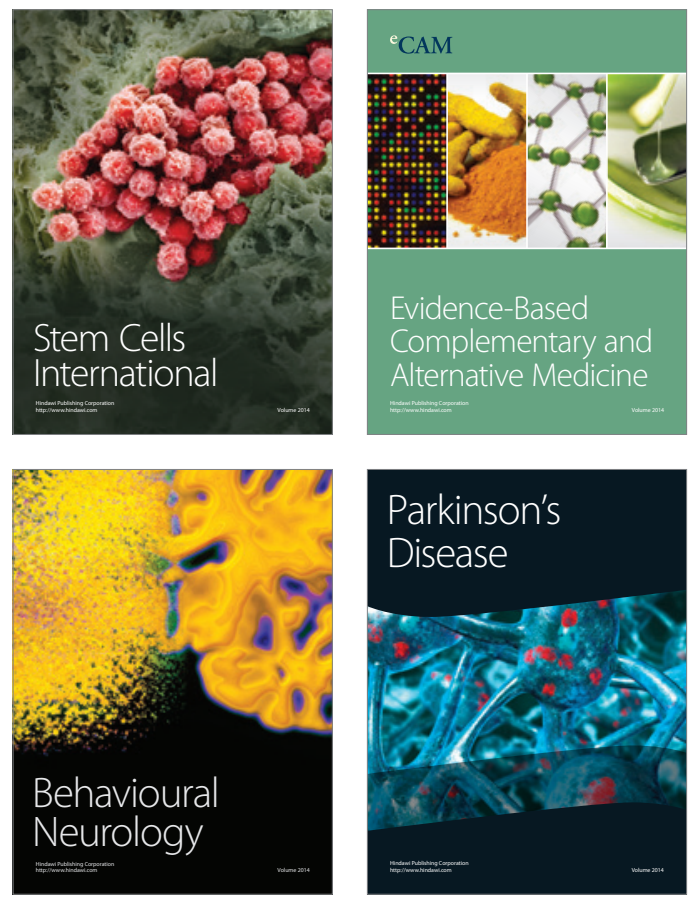
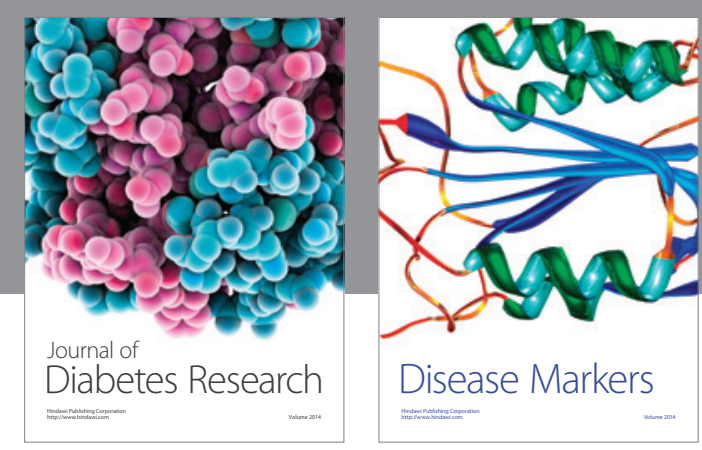

Disease Markers
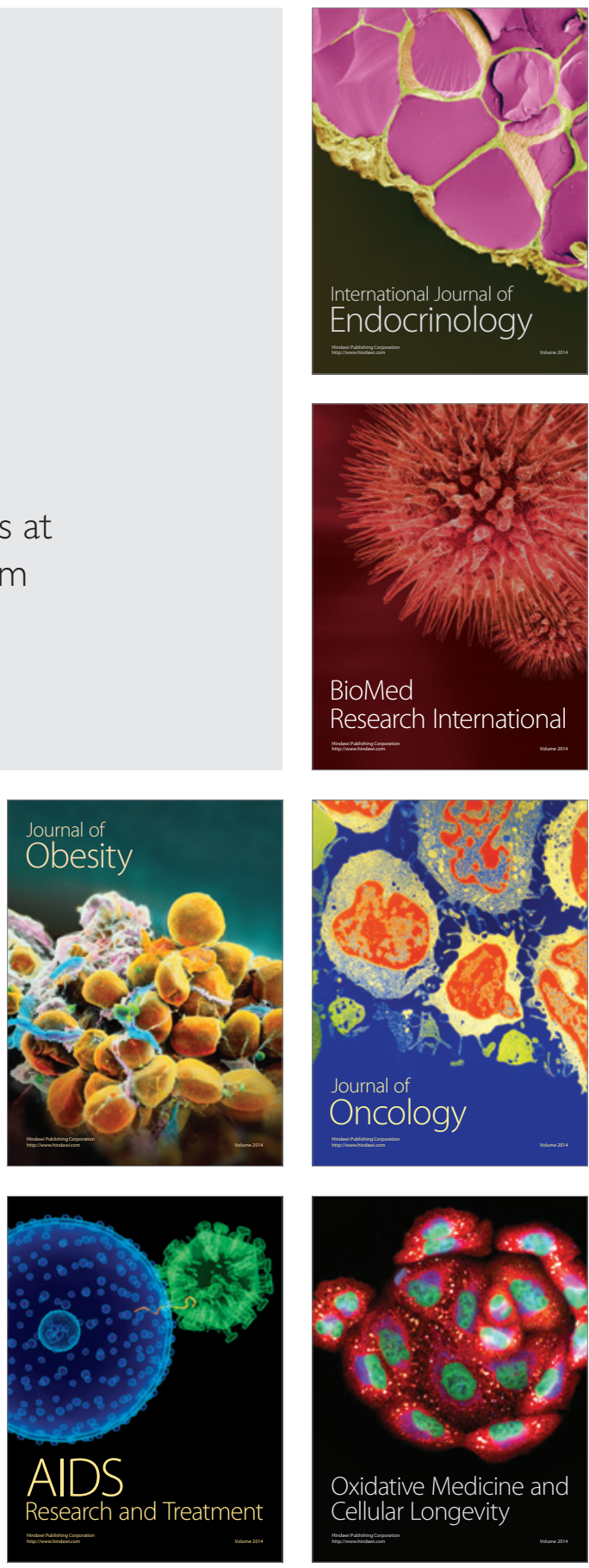